\title{
Migration and Transformation Characteristics of Niclosamide in a Soil-Plant System
}

\author{
Cui Luo, ${ }^{\dagger}$ Yiyang Huang, ${ }^{\ddagger}$ Donggen Huang, ${ }^{*}{ }^{\dagger} \odot$ Miao Liu, ${ }^{\dagger}$ Wei Xiong, ${ }^{\dagger}$ Qin Guo, ${ }^{\dagger}$ and Tianzi Yang
}

${ }^{\dagger}$ School of Resources Environment and Chemical Engineering, Nanchang University, Nanchang, Jiangxi 330031, China

${ }^{\ddagger}$ College of Environmental Science and Engineering, Beijing Forestry University, Beijing 100083, China

\section{Supporting Information}

ABSTRACT: The molluscicide niclosamide is found in most of the wetlands of China. The migration and transformation pathways, and degradation kinetics of niclosamide in the plant-soil system was analyzed by with the use of potting experiment. Experimental results showed that degradation of niclosamide in rhizosphere soil fit the first-order kinetics, and microorganisms played an important role in the degradation of niclosamide. It was found that niclosamide degrades to form a series of aromatic intermediate products both in soil and plants. Niclosamide could be absorbed from soil to plant by the root and then migrate to the stem. At an initial concentration of niclosamide of $2.11 \mathrm{mg} \cdot \mathrm{kg}^{-1}$ in soil, the maximum residue of niclosamide in Artemisia somai aerial was $2.47 \mathrm{mg} \cdot \mathrm{kg}^{-1}$ after 10 days of cultivation. This value is close to the pollution maximum residue limit $\left(3 \mathrm{mg} \cdot \mathrm{kg}^{-1}\right)$ in rice, and niclosamide and its intermediates can remain about 43 days in plants. The experimental results demonstrate that the use of niclosamide in wetlands would have some risk in edible plants and was harmful for human health.

\section{INTRODUCTION}

Niclosamide was the most efficient drug used in oncomelania control, and was designed to kill schistosome cercaria, miracidium, and tapeworm. Approximately $200 \mathrm{t}$ of niclosamide was added directly to Poyang Lake (China), and its degradation totally depends on natural methods such as diffusion-migration, hydrolysis, ${ }^{1,2}$ photolysis, ${ }^{3,4}$ and biodegradation. ${ }^{5,6}$ Although the use of niclosamide as a management strategy has been highly effective at controlling oncomelania, niclosamide was toxic to fish and other aquatic organisms. ${ }^{7,8}$ Meanwhile, niclosamide could inhibit the growth of hydrophytes especially phytoplankton. ${ }^{9}$ For example, niclosamide could lead to the poisoning death of grass carp, chub, and ostracean. ${ }^{10,11}$ For this reason, there is a need to better understand the fate and transformation products in lacustrine shoal.

The fate of niclosamide degradation in the natural environmental is not fully understood, but there were a few researches about it. Sediment soil has the strong ability to absorb niclosamide, and the organic content and clay composition in soil had significant impact on niclosamide absorbance. ${ }^{1}$ Niclosamide could diffuse and migrate in soil by water leaching. ${ }^{12}$ Niclosamide degrades through chemical reaction ${ }^{1,13}$ and photolysis $^{3,14,15}$ in lacustrine shoal. Biodegradation is the main path of niclsoamide degradation in the natural environment as aerobiotic and anaerobic naturalized microorganisms have a high capability of degrading niclosamide. ${ }^{16}$ Graebing found that niclosamide could hydrolyze to generate 5-chlorosalicylic acid and 2-chloro-4-nitroaniline under the effect of light; ${ }^{3}$ niclosamide in sediment could also convert to 2-chloro-4-nitroaniline and 5-chlorosalicylic acid whether in aerobic or anaerobic conditions. $^{16}$

At present, the major research about niclosamide was the lasting active time and toxicity in soil or water environment, there was little research in the literature about the migration and transformation of niclosamide in a plant-soil system. Research on this topic would benefit our understanding the degradation fate and pathway of niclosamide in natural environment.

The objective of this work was to study the degradation kinetics and pathway of niclosamide in a soil-plant system, and provide the degradation mechanism and risk data for niclosamide use. A plot model was used to simulate the degradation and transformation of niclosamide in the lacustrine shoal plant-soil system.

\section{RESULTS AND DISCUSSION}

Characterize of Niclosamide Degradation in Rhizosphere Soil. Degradation Kinetic of Niclosamide in Different Rhizosphere Soil. The residual concentration of niclosamide in carex, Artemisia somai rhizosphere soil, and blank control soil after the plants were potted was analyzed by HPLC, and the test results were shown in Figure 1. It can be seen from Figure 1 that the removal rate of niclosamide in three soil samples was the fastest in the first 3 days after the plant was potted, and the removal rate of niclosamide in carex, Artemisia somai rhizosphere soil, and blank soil was $27.7 \%, 23.1 \%$, and $18.9 \%$, respectively. The recovery rate of niclosamide added in soil was $88.65 \%-92.85 \%$, and standard deviation was 3.45\%-6.74\% (see the Supporting Information). Three days later, the changes in the removal rate of niclosamide in the three soils showed the nonmonotone change of "slow-gradual acceleration-gradual deceleration”. The removal rate of niclosamide in carex, Artemisia somai rhizosphere soil, and blank soil after culturing for 43 days was $79.8 \%, 73.6 \%$, and $67.9 \%$, respectively.

According to Hamaker's pesticide and other organic compound degradation dynamic model, ${ }^{17}$ the fitting process of the niclosamide residual concentration in carex, Artemisia somai

Received: January 12, 2018

Accepted: February 14, 2018

Published: February 27, 2018 


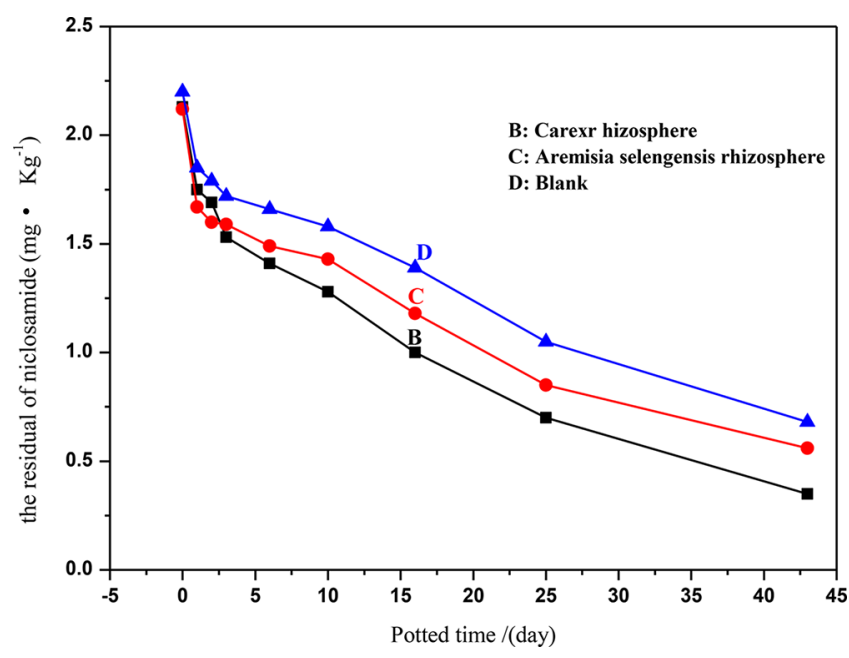

Figure 1. Relationship between niclosamide residual concentration and potted time in rhizosphere soil.

rhizosphere soil, and blank soil after the potted plants was carried out, and the results were shown in Figure 2. It could be seen from

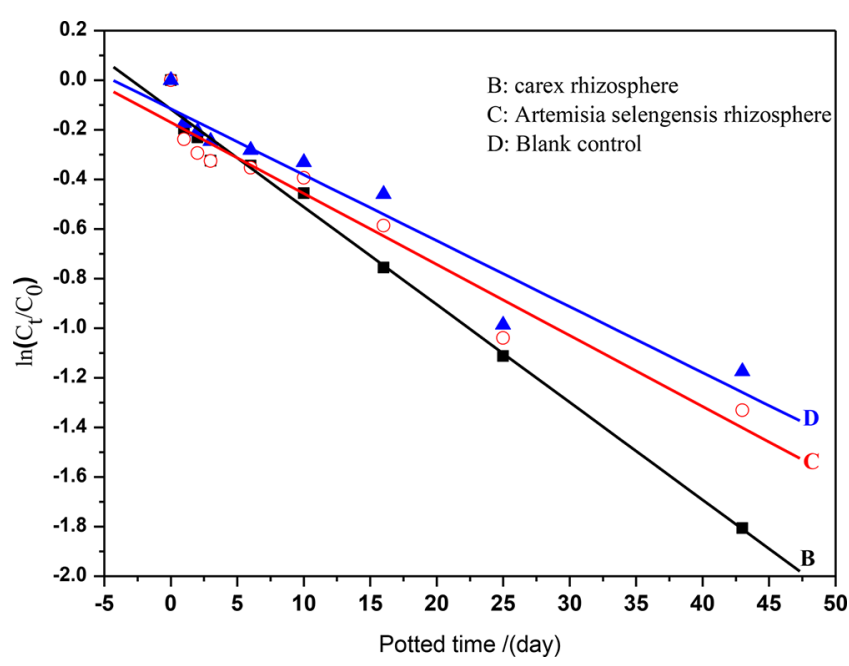

Figure 2. Fitting curves of niclosamide degradation kinetics in soil samples.

Figure 2 that the removal rate of niclosamide in carex, Artemisia somai rhizosphere soil, and blank soil met the kinetics characteristics of pseudo-first-order, and there was a good linear relationship between the logarithm of residual concentration $(y)$ and degradation time $(t)$ in carex, Artemisia somai rhizosphere soil, and blank soil. The parameters of the degradation kinetic equation were shown in Table 1 . It could be seen that the degradation rate constant of niclosamide in carex, Artemisia

Table 1. Degradation Kinetics of Niclosamide in Different Soil Samples

\begin{tabular}{|c|c|c|c|c|}
\hline soil samples & $\begin{array}{l}\text { dynamical } \\
\text { equation }\end{array}$ & $\begin{array}{c}\mathrm{C}_{0} \\
(\mathrm{mg} / \mathrm{kg})\end{array}$ & $k$ & $R$ \\
\hline carex rhizosphere & $\begin{array}{c}y=-0.1169- \\
0.0394 x\end{array}$ & 2.13 & -0.0394 & 0.9946 \\
\hline $\begin{array}{l}\text { Artemisia somai } \\
\text { rhizosphere }\end{array}$ & $\begin{array}{c}y=-0.1664- \\
0.0270 x\end{array}$ & 2.12 & -0.0270 & 0.9803 \\
\hline blank control soil & $\begin{array}{c}y=-0.1121- \\
0.0248 x\end{array}$ & 2.20 & -0.0248 & 0.9881 \\
\hline
\end{tabular}

somai rhizosphere soil, and blank soil was $-0.0394,-0.0270$, and -0.0248 , and the half-life of niclosamide in carex, Artemisia somai, and blank rhizosphere soil was 14.6, 18.3, and $21.7 \mathrm{~d}$, respectively.

Combined Table 1 and Figure 2, carex rhizosphere exhibited a faster degradation of niclosamide than Artemisia somai rhizosphere, and the degradation rate in plant rhizosphere was faster than that in the blank control soil. Especially, the removal rate of niclosamide in soil was quick in the first 3 days after the plants were potted, which might be caused by the hydrolytic reaction of niclosamide in moist soil in the initial phase. The results showed that these two plants had a catalytic effect on the degradation of niclosamide, and the effect of carex was greater than that of Artemisia somai.

Transform Features of Niclosamide in the Rhizosphere Soil. Because intermediate products may be produced by the degradation of niclosamide in the natural environment, HPLC chromatography was used to qualitatively analyze the parent and possible degradation products of niclosamide. The limit of detection (LOD) was $0.3 \mu \mathrm{g} \cdot \mathrm{kg}^{-1}$ and the limit of quantitation (LOQ) was $0.9 \mu \mathrm{g} \cdot \mathrm{kg}^{-1}$ (see the Supporting Information).

The target products were qualitatively analyzed according to peak retention time of the single standard sample, mixed standard sample, and test sample in the same HPLC analysis conditions. HPLC spectrograms of a single standard sample and mixed standard sample were shown in Figure 3. Figure 3 indicated that niclosamide and its natural degradation middle products such as 2,5-dihydroxy benzoic acid, 2-chloro-4nitroaniline, 2-chloro-4-nitrophenol, aminoniclosamide, 5-chlorosalicylic acid, and niclosamide were well separated in the set instrumental analysis conditions. In addition to 5-chlorinated salicylic acid, other substances had good response values when the light of the detection channel was $330 \mathrm{~nm}$. 5-Chlorosalicylic acid had good response value when the light of the detection channel was $285 \mathrm{~nm}$. The retention time of 2,5-dihydroxy benzoic acid, 2-chloro-4-nitroaniline, 2-chloro-4-nitrophenol, aminoniclosamide, 5-chlorosalicylic acid, and niclosamide was $3.55,6.70,7.81,10.21,11.46$, and $12.23 \mathrm{~min}$, respectively.

In the soil of carex, Artemisia somai rhizosphere, and blank control group, niclosamide was converted into many intermediate products. Figure 4 was the HPLC spectrogram of niclosamide degraded intermediate product in carex rhizosphere soil which was the sixth day after potting. It can be seen from Figure 4 that part of niclosamide was degraded into 2,5dihydroxy benzoic acid (3.48 $\mathrm{min})$, 2-chloro-4-nitroaniline (6.79 $\mathrm{min})$, 2-chloro-4-nitrophenol (7.89 $\mathrm{min})$, aminoniclosamide (10.40 $\mathrm{min})$, and 5-chloro salicylic acid (11.43 $\mathrm{min})$; however, due to the analysis conditions, there were also some compounds, the retention times of which were $2.57,4.33,9.56,15.38$, and $15.77 \mathrm{~min}$, that were not qualitatively analyzed.

The parent and possible intermediate product of niclosamide natural degradation were tested by HPLC-MS/MS, and the results were shown in Figure 5. Figure 5 peaks $A$ and $B$ comprised the total ion current (TIC) and indicated that there was a number of intermediates generated during niclosamide natural degradation, and more intermediates were found in plant rhizosphere soil than in the blank control soil. The analysis results of TIC by MS/MS were shown in Figure 5C. Those results showed that there were several molecular ion peaks such as $124.89,141.86,143.86,152.89,170.88,172.94,173,297.15$, 325.18 , etc. detected at $2.845,3.390,3.430,2.570,7.037,3.524$, $5.769,10.735$, and $12.325 \mathrm{~min}$ in TIC, respectively. The possible molecular formula and structural formula were deduced in Table 


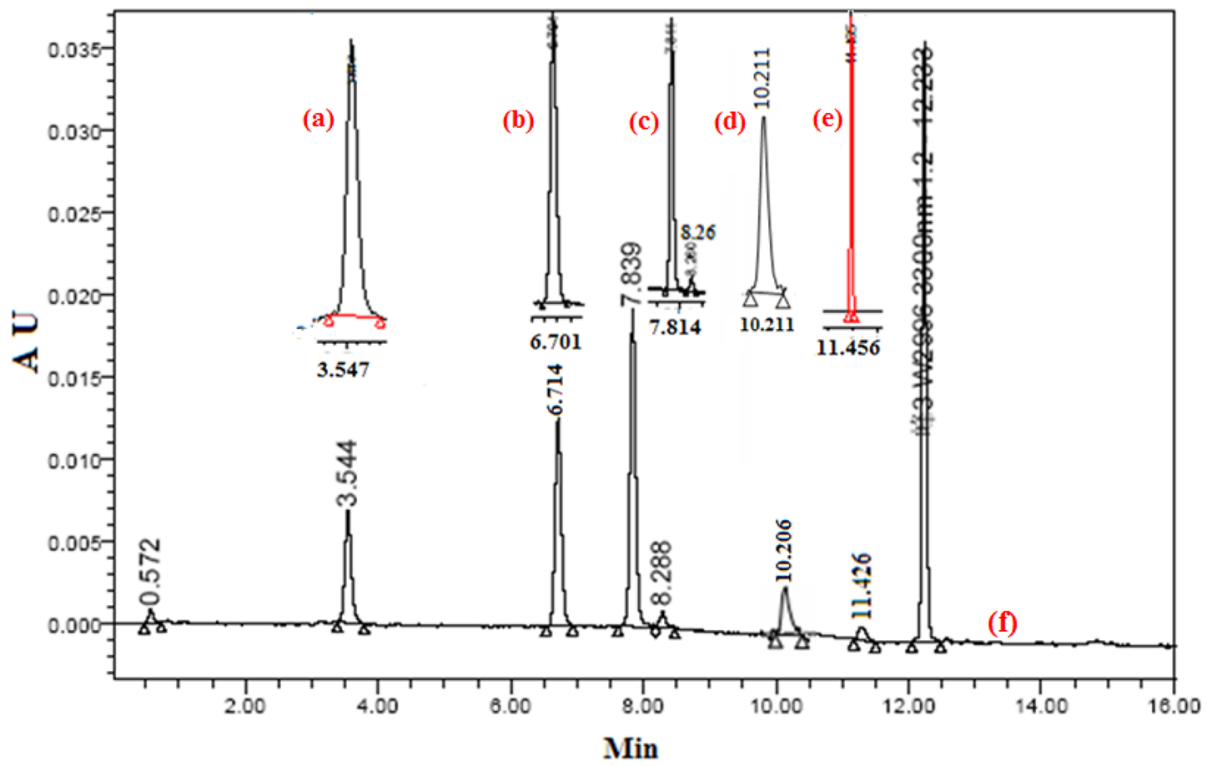

Figure 3. HPLC spectrogram of niclosamide and possible intermediate standard samples: (a) 2,5-dihydroxy benzoic acid (5 mg. $\left.\mathrm{L}^{-1}\right)$, (b) 2-chloro-4nitroaniline $\left(5 \mathrm{mg} \cdot \mathrm{L}^{-1}\right),\left(\right.$ c) 2-chloro-4-nitrophenol $\left(5 \mathrm{mg} \cdot \mathrm{L}^{-1}\right)$, (d) aminoniclosamide $\left(2 \mathrm{mg} \cdot \mathrm{L}^{-1}\right)$, (e) 5-chlorosalicylic acid $\left(5 \mathrm{mg} \cdot \mathrm{L}^{-1}\right.$, detection channel $285 \mathrm{~nm})$, (f) mixed standard sample $\left(5 \mathrm{mg} \cdot \mathrm{L}^{-1}\right.$, respectively).

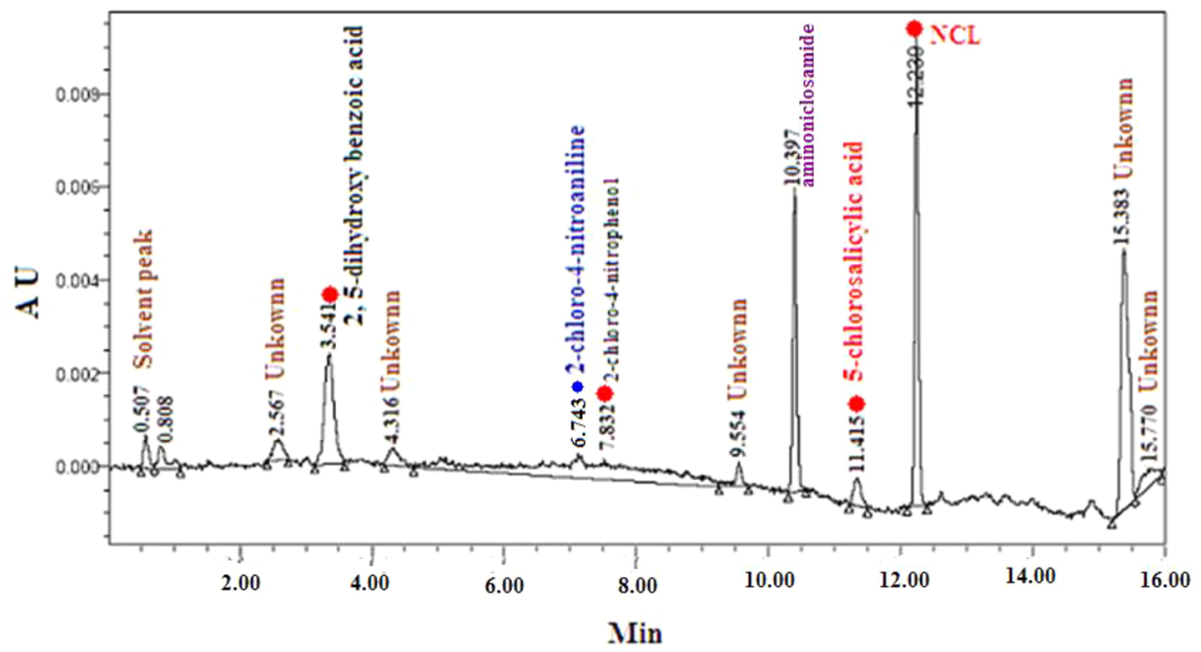

Figure 4. Degradation products HPLC spectrogram of Niclosamide in rhizosphere soil after carex pot culture for the sixth day.

2. The results of Table 2 illustrated that some hydrolysate such as 5-chloro salicylic acid and 2-chloro-4-nitroaniline were produced, some reduction products such as aminoniclosamide and 2-chloro-p-phenylenediamine were produced, and some oxidative degradation products such as 2,5-dihydroxy benzoic acid and hydroxyhdroquinone were produced.

Combined HPLC and HPLC-MS/MS analysis results indicated that niclosamide would be degraded into a series of intermediate products such as aminoniclosamide, 5-chlorosalicylic acid, 2-chloro-4-nitroaniline, 2-chloro-4-nitrophenol, 2,5-dihydroxy benzoic acid, 2-chloro- $p$-phenylenediamine, hydroxyhdroquinone and 2-chlor-4-hydroxyphenol, etc., in rhizosphere soil.

Effects of Microorganism Populations on Niclosamide Degradation in Rhizosphere Soil. Dynamic Change of Microbial Population in Soil. The population dynamic change of bacteria, fungi, and actinomycetes in the rhizosphere soil of carex, Artemisia somai after potting, and the control group soil were shown in Figure 6. The results of Figure 6 indicated that bacteria was the microorganisms found the most in the rhizosphere soil of carex, Artemisia somai, and the control group soil, followed by fungi and then actinomycetes, which was consistent with the distribution of microorganisms in soil. In the course of the entire cultivation, the amount of microorganisms in the plant rhizosphere increased with the last cultivation time, which was faster than that in control group, thus showing plant root exudates could promote microbial growth. The population of bacteria in the plant rhizosphere of carex and Artemisia somai reached a peak at the 16th day of cultivation, and actinomycetes population reached the highest point at the 25th day of cultivation, which is consistent with the half-life of niclosamide degradation in the two kinds of plant rhizosphere soil (Table 1).

Relationships between Niclosamide Residues and Microbial Population in Rhizosphere Soil. There was a good correspondence between the niclosamide residues and the population of bacteria, fungi, and actinomycetes in the plant 


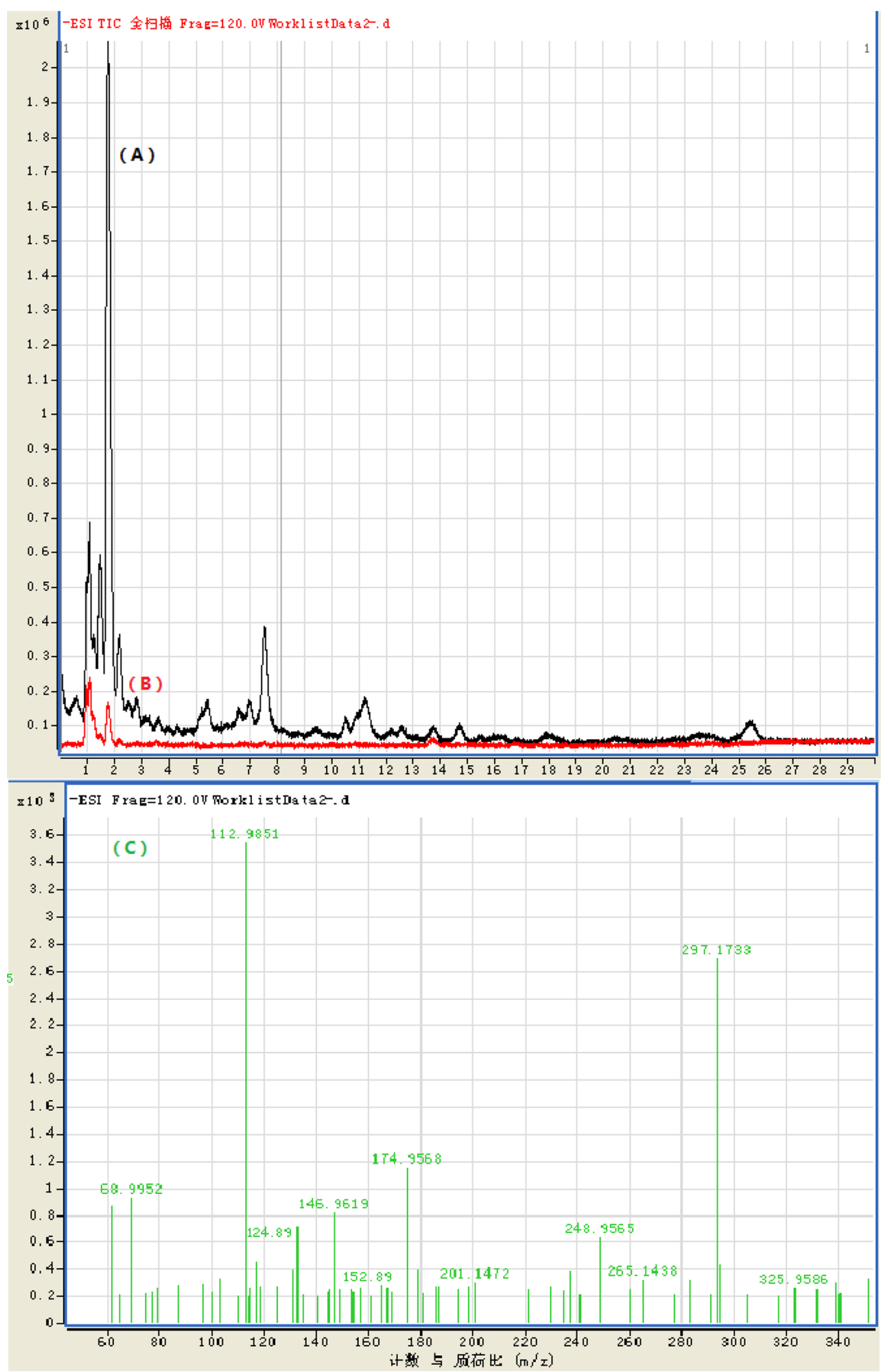

Figure 5. HPLC-MS/MS analysis sketch of niclossmide and its degradation intermediate products in carex soil. HPLC-MS/MS analysis sketch of niclosamide and its degradation intermediate products in carex soil: (A) TIC of black control soil; (B) TIC of carex rhizosphere soil; (C) molecular ion peak.

rhizosphere soil. Results were shown in Figure 7. Figure 7 demonstrated that niclosamide residues in carex and Artemisia somai soil were significantly negatively correlated with the amount of three kinds of microbes $(P<0.05)$. Namely, in two kinds of plant rhizosphere soil, the greater was the number of microorganisms, the less was the residues of niclosamide. Figure 
Table 2. HPLC-MS/MS Analysis Results of Niclosamide Degradation Possible Intermediate Products in Potting Soil and Artemisia Somai Sample

\begin{tabular}{|c|c|c|c|c|c|c|}
\hline \multirow{2}{*}{$\begin{array}{l}\text { Serial } \\
\text { number }\end{array}$} & \multicolumn{2}{|c|}{ Potting soil } & \multicolumn{2}{|c|}{$\begin{array}{l}\text { potting artemisia } \\
\text { Selengensis }\end{array}$} & \multirow{2}{*}{$\begin{array}{l}\text { Possible } \\
\text { formulas }\end{array}$} & \multirow{2}{*}{$\begin{array}{l}\text { Possible structural } \\
\text { formula }\end{array}$} \\
\hline & Ion peak & $\begin{array}{l}\text { Peak } \\
\text { Time/min }\end{array}$ & Ion peak & $\begin{array}{c}\text { Peak } \\
\text { Time/min }\end{array}$ & & \\
\hline 1 & 124.8908 & 2.854 & 124.9793 & 7.280 & $\mathrm{C}_{6} \mathrm{H}_{6} \mathrm{O}_{3}$ & \\
\hline 2 & 141.8682 & 3.390 & 141.9617 & 5.260 & $\mathrm{C}_{6} \mathrm{H}_{7} \mathrm{ClN}_{2}$ & \\
\hline 3 & 143.8658 & 3.340 & 143.9267 & 5.439 & $\mathrm{C}_{6} \mathrm{H}_{5} \mathrm{ClO}_{2}$ & \\
\hline 4 & 152.8969 & 2.570 & 152.9964 & 5.349 & $\mathrm{C}_{7} \mathrm{H}_{6} \mathrm{O}_{4}$ & \\
\hline 5 & 170.8845 & 7.037 & 170.9124 & 3.847 & $\mathrm{C}_{7} \mathrm{H}_{5} \mathrm{ClO}_{3}$ & \\
\hline 6 & 172.9415 & 3.524 & 172.9525 & 1.351 & $\mathrm{C}_{6} \mathrm{H}_{4} \mathrm{CINO}_{3}$ & \\
\hline 7 & 173.0118 & 5.769 & 173.0136 & 9.589 & $\mathrm{C}_{6} \mathrm{H}_{5} \mathrm{ClN}_{2} \mathrm{O}_{2}$ & \\
\hline 8 & 297.1528 & 10.735 & 297.1470 & 5.123 & $\mathrm{C}_{13} \mathrm{H}_{10} \mathrm{Cl}_{2} \mathrm{~N}_{2} \mathrm{O}_{2}$ & \\
\hline 9 & 325.183 & 12.325 & 324.9716 & 9.996 & $\mathrm{C}_{13} \mathrm{H}_{8} \mathrm{Cl}_{2} \mathrm{~N}_{2} \mathrm{O}_{4}$ & \\
\hline
\end{tabular}

7 also showed that the microorganisms such as bacteria, fungi, and actinomycetes played an important role in the degradation of niclosamide in rhizosphere soil.

The Migration and Transformation Characteristics of Niclosamide in the Soil-Plant System. The Characteristics of Niclosamide Residue in Plants. Niclosamide residue in plants at different times were quantitatively analyzed by using HPLC after plant potting; the results were shown in Table 3. The analysis results in Table 3 showed that the maximum niclosamide residue in the aerial and root part of Artemisia somai were 2.47 $\mathrm{mg} \cdot \mathrm{kg}^{-1}$ and $0.99 \mathrm{mg} \cdot \mathrm{kg}^{-1}$ after cultivation for 10 and 2 days, respectively. The maximum niclosamide residue in aerial and root part of carex were $1.7 \mathrm{mg} \cdot \mathrm{kg}^{-1}$ and $1.0 \mathrm{mg} \cdot \mathrm{kg}^{-1}$ after cultivation for 6 days. Niclosamide mainly existed in the aerial part of Artemisia somai, and the concentration of niclosamide in the aerial part decreased rapidly after 10 days. The trend of niclosamide in carex was the same as in Artemisia somai, the differences were that the concentration decreased rapidly after 2 days. In the two plants, niclosamide residue concentration in the aerial part was higher than that in the root part, and decreased rapidly in the aerial part. The residue of niclosamide could not be detected in carex aerial part after 16 days, which meant that the niclsoamide degradation was nearly completed.

The Migration and Transformation Characteristics of Niclosamide within Plants. Niclosamide and its possible intermediate product in a plant interior at different times were qualitatively and quantitatively analyzed by using the retention time comparison method and quantitative test by normal curve method of HPLC after the plant was potted. The results were shown in Figure 8 and Table 3. It can be seen from Figure 8 that a variety of extractive products would be produced during the preparation process of blank Artemisia somai analysis sample; potted 6 days later, the ground part of Artemisia somai would produce 2,5-dihydroxy benzoic acid (3.48 $\mathrm{min})$, 2-chloro-4nitroaniline (6.79 $\mathrm{min}$ ), 2-chloro-4-nitro phenol (7.89 $\mathrm{min})$, 5chloro salicylic acid (11.43 $\mathrm{min}$ ) and niclosamide residues (12.27 $\min )$; However, due to the analysis conditions, there were also some compounds whose retention time was 4.02, 4.49, 7.52, $8.79,13.43$ and $14.72 \mathrm{~min}$ that were not identified. The results of Table 3 indicated that the concentration of the intermediate products which were qualitatively detected by niclosamide 

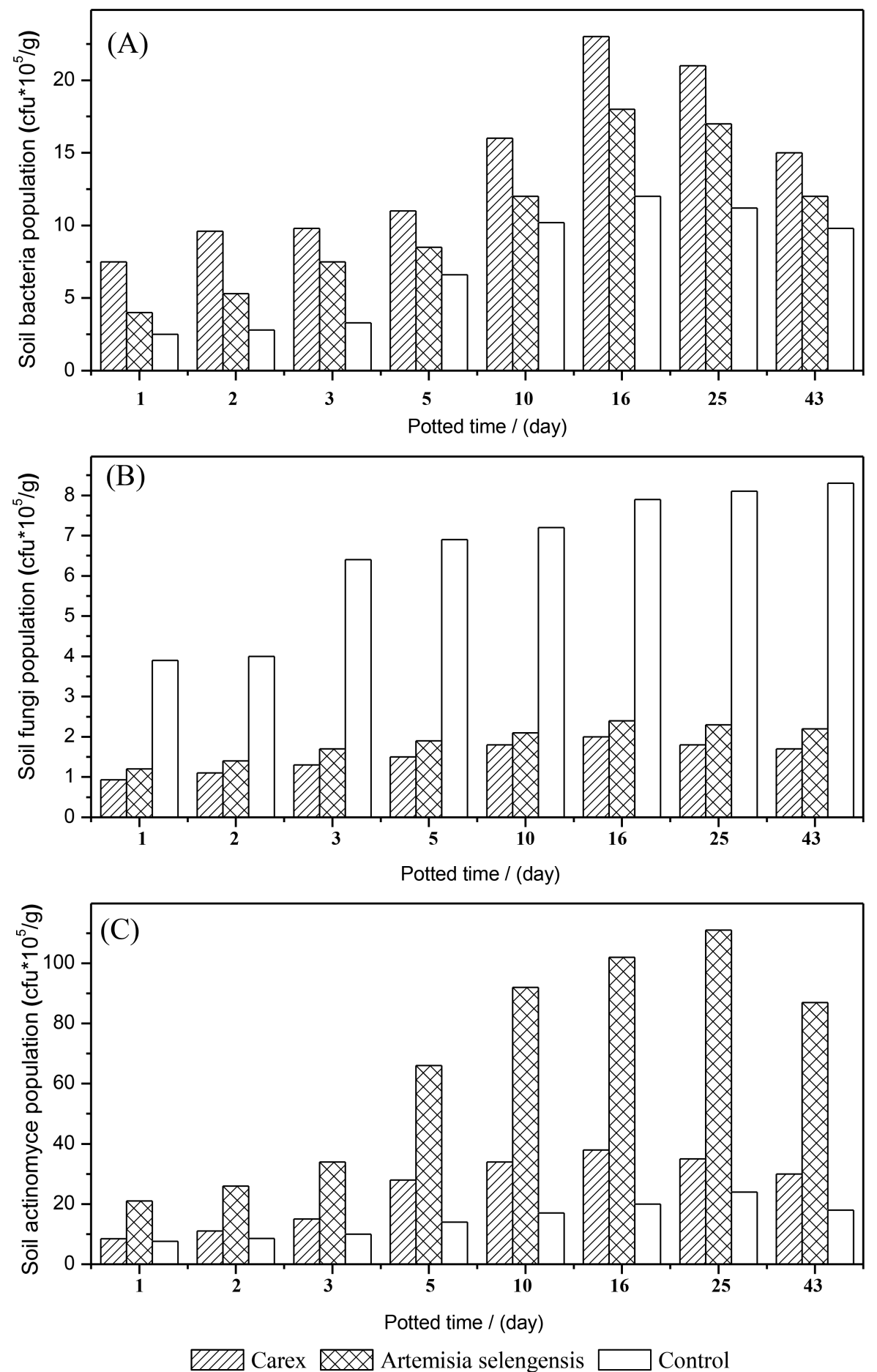

Figure 6. Population of microorganism in rhizosphere soil. Dynamic change of microorganism population in soil: (A) bacteria; (B) fungi; (C) actinomycetes.

degraded in plants appeared to change dynamically with time, and the concentration decreased until it disappeared entirely.

The combined analysis results show that niclosamide would be degraded into a series of intermediate products such as aminoniclosamide, 5-chloro salicylic acid, 2-chloro-4-nitroaniline, 2-chloro-4-nitro phenol, 2, 5-dihydroxy benzoic acid, 2chloro-p-phenylenediamine, hydroxyhdroquinone and 2-chlor4-hydroxyphenol, etc.

The qualitative and quantitative analysis results of niclosamide and its possible intermediate product in the plant interior at different times indicated that niclosamide migrated from soil into the stem and leaf by plant roots, and transformed into a variety of intermediates through physical and chemical reaction under the action of plant enzymes in a potted plant-soil system. In this process, the hydrolysis reaction of niclosamide occurred under the action of plants' water, and produced 5-chloro salicylic acid and 2-chloro-4-nitroaniline; a redox reaction happened under the action of the plant enzymes and produced aminoniclosamide, 2chloro-p-phenylenediamine, 2,5-dihydroxy benzoic acid, and hydroxyhdroquinone, etc. 

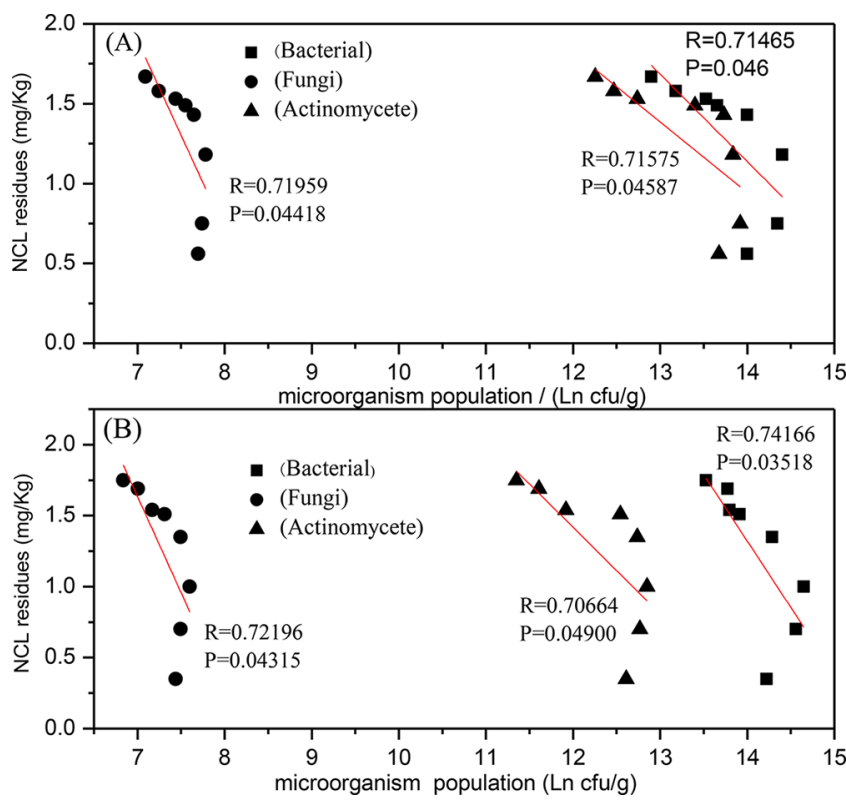

Figure 7. Relationships between niclosamide residues and microbial population in rhizosphere soil: (A) carex rhizosphere soil; (B) Artemisia somai rhizosphere soil.

The Migration and Transformation Mechanism of Niclosamide in Soil-Plant System. Comprehensive experimental results, in the soil-plant system show that niclosamide could be degraded in soil by physical, chemical, and biodegradation pathways under the action of water, microorganisms, and plant root secretions in soil, and produce 5chlorosalicylic acid, 2-chloro-4-nitroaniline, aminoniclosamide, 2-chloro- $p$-phenylenediamine, 2,5-dihydroxy benzoic acid, and hydroxyhdroquinone, etc (Table 3). Also, niclosamide migrated from soil into the stem and leaf by plant roots.

Niclosamide and its possible intermediate in the plant were degraded and produced 5-chloro salicylic acid, 2-chloro-4nitroaniline, aminoniclosamide, 2-chloro- $p$-phenylenediamine, 2,5-dihydroxy benzoic acid, etc., under the action of water and enzymes in plants (Table 3). Finally, the intermediate was metabolized into simple compounds such as $\mathrm{CO}_{2}$ and $\mathrm{H}_{2} \mathrm{O}$. On the basis of the results just mentioned, the migration and transformation mechanism of niclosamide in the soil-plant system was proposed and shown in Figure 9.

Risk Analysis of Edible Plants after the Use of Niclosamide. Author: The results of Table 2 indicated that niclosamide and it possible intermediate products (such as 5-chlorinated salicylic acid, 2-chlorine-4-nitroaniline, 2-chloro-4- nitrophenol, aminoniclosamide) would remain in the plant interior. According to previous studies, niclosamide was highly toxic to fish and amphibians, ${ }^{18,19}$ 5-chlorinated salicylic acid had an effect on mutation and carcinogenesis, ${ }^{20}$ 2-chlorine-4-nitroaniline could produce a lower level of mutation than niclosamide, ${ }^{21}$ and 2chlor-4-nitrophenol belongs to the high toxic organic compound, and its acute oral toxicity (LD50) to rats was $900 \mathrm{mg} \cdot \mathrm{kg}^{-1}$. Thus, we can conclude that there was a risk to edible plants when niclosamide was used.

The results of Table 3 showed that the concentrations of niclosamide residue and intermediate in plant material were changing with time, the maximum niclosamide residue in the aerial and root part of Artemisia somai was $2.47 \mathrm{mg} \cdot \mathrm{kg}^{-1}$ and 0.99 $\mathrm{mg} \cdot \mathrm{kg}^{-1}$, respectively. In the aerial and root part of carex the maximum niclosamide residue was $1.7 \mathrm{mg} \cdot \mathrm{kg}^{-1}$ and $1.0 \mathrm{mg} \cdot \mathrm{kg}^{-1}$. The maximum concentration of 2-chloro-4-nitrophenol in Artemisia somai was $0.065 \mathrm{mg} \cdot \mathrm{kg}^{-1}$ and was $0.032 \mathrm{mg} \cdot \mathrm{kg}^{-1}$ in carex which had a risk for rabbit. As for 2-chloro-4-aminophenol, the concentration was much higher than that of 2-chloro-4nitrophenol, the maximum concentration was $0.741 \mathrm{mg} \cdot \mathrm{kg}^{-1}$ in Artemisia somai and was $0.125 \mathrm{mg} \cdot \mathrm{kg}^{-1}$ in carex.

At present, China, United Nations Food and Agriculture Organization (UNFAO); America, and the European Union (UN) do not formulate the maximum residue limit (MRL) of niclosamide in edible plant. According to the MRL of niclosamide in rice formulated by China, which is $3.0 \mathrm{mg}$. $\mathrm{kg}^{-1}, 21$ the maximum residue of niclosamide in the edible plant Artemisia somai approached the standard values, which constitutes some risks. Moreover, the concentration of niclsoamdie residue in Artemisia somai was still high after 16 days, which demonstrated that niclosamide remained a long time

Table 3. Concentration of Niclosamide and Its Degraded Intermediate in Plants $\left(\mu \mathrm{g}^{\mathrm{kg}} \mathrm{g}^{-1}\right)$

\begin{tabular}{|c|c|c|c|c|c|c|}
\hline & plant parts & $\mathrm{HA}^{a}(3.544 \mathrm{~min})$ & $2 \mathrm{C}^{2} \mathrm{NBA}^{b}(6.714 \mathrm{~min})$ & $2 \mathrm{C} \mathrm{NBP}^{c}(7.839 \mathrm{~min})$ & $5 \mathrm{CA}^{d}(11.456 \mathrm{~min})$ & $\mathrm{NCL}^{e}(12.233 \mathrm{~min})$ \\
\hline \multirow[t]{2}{*}{$\mathrm{AS}^{f}$ (2nd day) } & root part & 529 & 703.0 & 5.23 & 1.6 & 840 \\
\hline & aerial part & 137.5 & 626.2 & 8.0 & 9.6 & 2410 \\
\hline \multirow[t]{2}{*}{ AS (sixth day) } & root part & 92.2 & 165.4 & 3.50 & 3.6 & 910 \\
\hline & aerial part & 423.6 & 171.4 & 57.5 & 4.6 & 2450 \\
\hline \multirow[t]{2}{*}{ AS (10th day) } & root part & 61.8 & 596.2 & 8.16 & 4.3 & 990 \\
\hline & aerial part & 253.4 & 741.2 & 65.43 & 12.0 & 2470 \\
\hline \multirow[t]{2}{*}{ AS (16th day) } & root part & $\mathrm{ND}^{g}$ & 39.9 & 15.00 & $\mathrm{ND}$ & 950 \\
\hline & aerial part & ND & 322.3 & 3.67 & ND & 1220 \\
\hline \multirow[t]{2}{*}{ carex (2nd day) } & root part & ND & 4.80 & 6.0 & 0.3 & 500 \\
\hline & aerial part & ND & 125.5 & 9.8 & 5.4 & 1670 \\
\hline \multirow[t]{2}{*}{ carex (6th day) } & root part & ND & $\mathrm{ND}$ & 5.7 & 3.8 & 1000 \\
\hline & aerial part & ND & 48.64 & 32.0 & 8.5 & 1700 \\
\hline \multirow[t]{2}{*}{ carex (10th day) } & root part & 466.0 & 20.4 & 10.7 & 1.8 & 650 \\
\hline & aerial part & 208.8 & 77.2 & 7.5 & 1.9 & 590 \\
\hline \multirow[t]{2}{*}{ carex (16th day) } & root part & 30.3 & 14.1 & 8.9 & 1.2 & 390 \\
\hline & aerial part & 0.63 & 0.22 & 0.1 & $\mathrm{ND}$ & ND \\
\hline
\end{tabular}

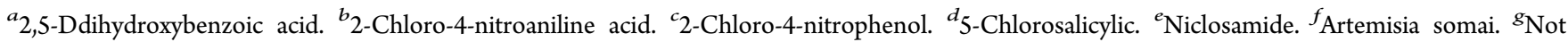
detected. 


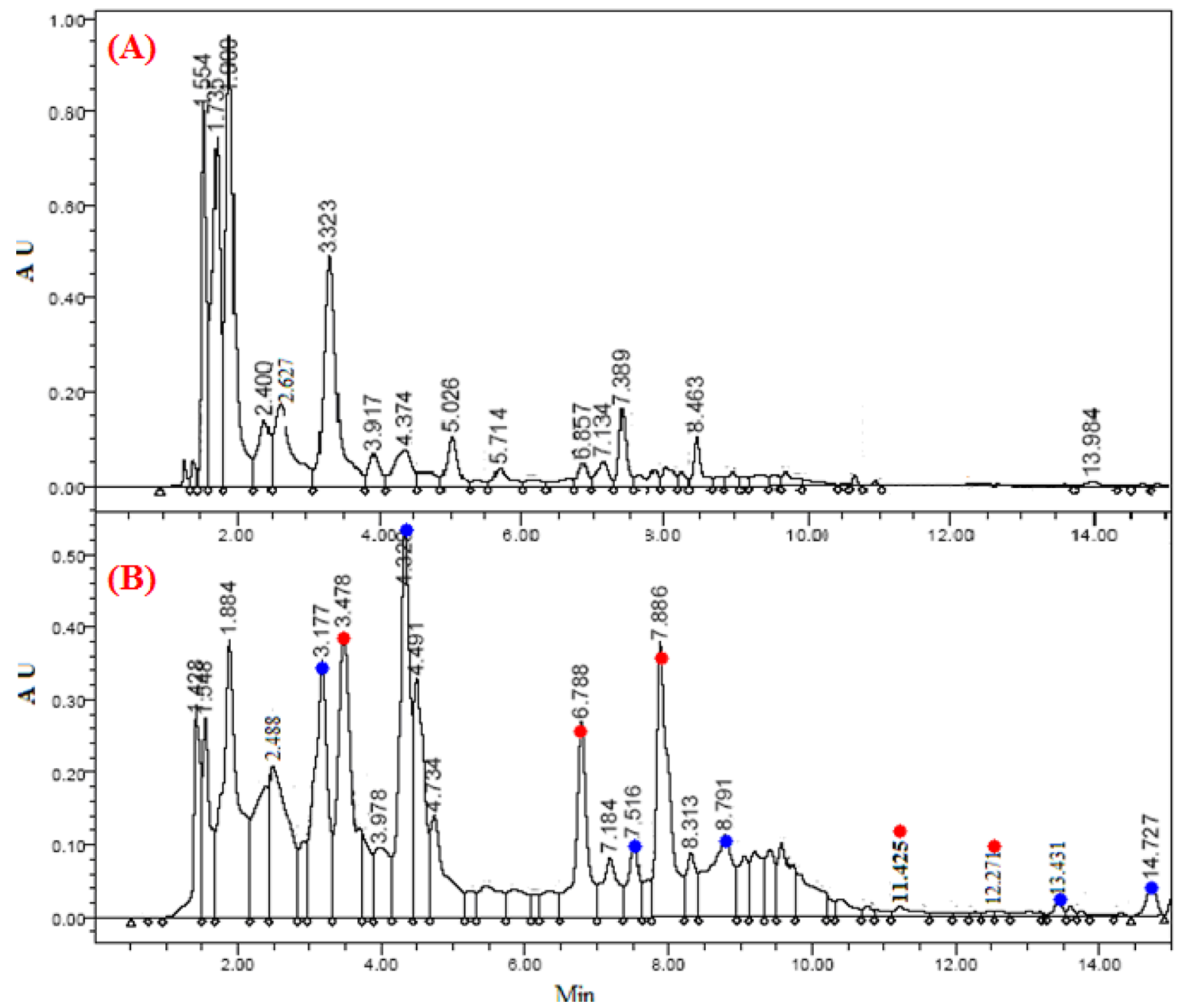

Figure 8. HPLC spectra of niclosamide residue and possible intermediate in Artemisia somai: (A) blank, Artemisia somai overground part; (B) Cultured for 6 days: Artemisia somai overground part.

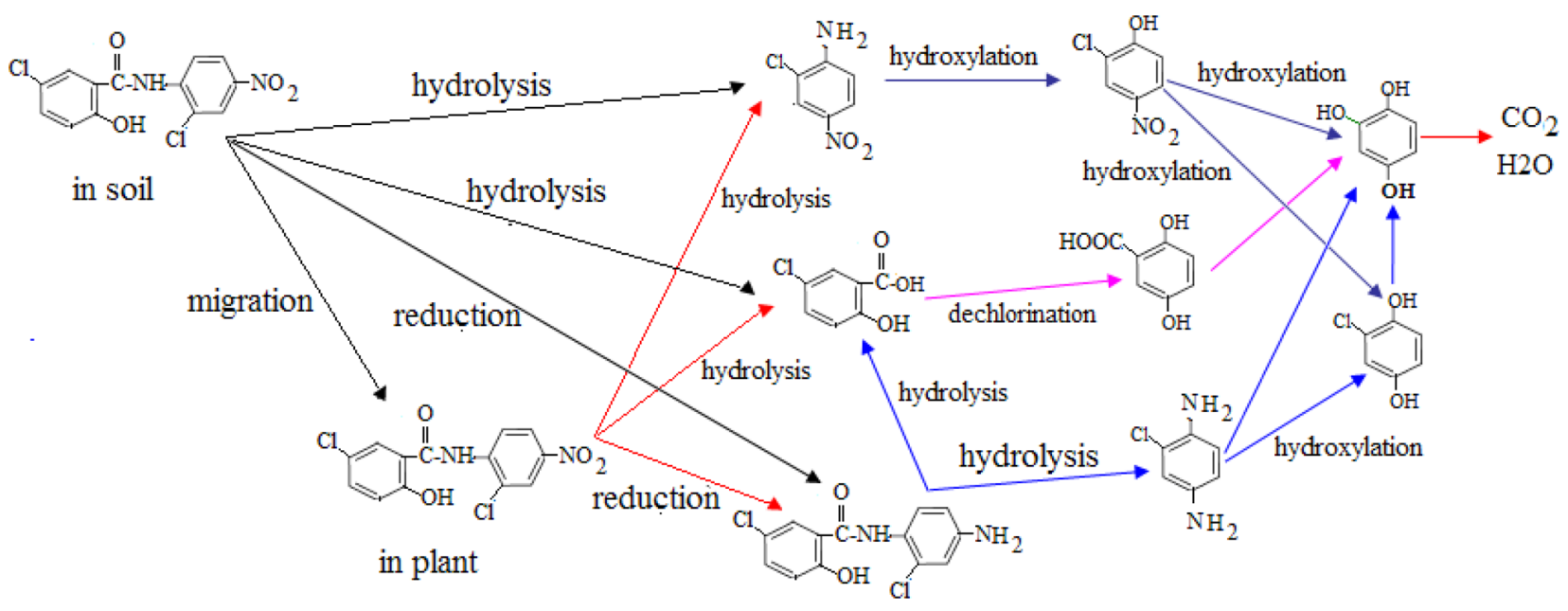

Figure 9. Possible migration and transformation mechanism of niclosamide in the soil-plant system.

in Artemisia somai. Consequently, it was concluded that niclsaomdie could pose a risk to edible plants.

\section{CONCLUSION}

In this study, we had found that the degradation of niclosamide in rhizosphere soil was in accordance with the first-order kinetic equation. Carex and Artemisia selengensis rhizosphere could promote the degradation of niclosamide in soil, and carex showed a greater rate of degradation. In the plant-soil system, the presence of a grater amount of microorganism resulted in less niclosamide residue, which indicated that microorganisms had sn important contribution to the degradation of niclosamide. 
Moreover, we analyzed the microbial species in each rhizosphere soil and found that the main microorganism in the carex rhizosphere was bacteria, was actinomycetes in Artemisia selengensis rhizosphere, and was fungi in the control. This indicated that a plant could adjust the physical and chemical properties of the soil, and bacteria were more conductive niclosamide degradation. In the soil and plant interior, niclosamide could degrade to a series of aromatic products under the action of hydrolysis, microorganisms, and plant enzymes, etc. The niclosamide residue and its degradation products remain a long time in plants, which may pose a risk for the edible plant.

\section{MATERIALS AND METHODS}

Materials. Niclosamide (CAS: 50-65-7) was supplied from Chengdu Grecian chemical technology Co. Ltd., niclosamide salt wettable powder (CAS: 1420-04-8) was supplied from Anhui dongsheng pharmaceutical Co. Ltd., 2,5-dihydroxybenzoic acid (CAS: 490-79-9), 2-chloro-4-nitroaniline (CAS: 121-87-9), 2chloro-4-nitrophenol (CAS: 619-08-9), aminoniclosamide (CAS: 50-65-7), 5-chloro-2-hydroxybenzoic acid (CAS: 32114-2) were supplied from Shandong Xiya reagent research center. All reagents were of high purity grade and used as received. Experiment soil was taken from the lacustrine shoal of Nanji Mountain, Nanchang, China. Experiment plants were the typical plant (Artemisia somai and carex spp.) of Nanji Mountain in high flow season.

Bonsai Preparation. Soil for experiment was air-dried and passed through $3 \mathrm{~mm}$ sieves. The niclosamide methanol solution was sprayed evenly to the soil and mixed well with the soil until the methanol completely vaporized. The concentration of niclosamide was $2.11 \mathrm{mg} \cdot \mathrm{kg}^{-1}$ in the suspended soil. The soil was subpacked evenly in 24 flowerpots (diameter, $15 \mathrm{~cm}$; height, $20 \mathrm{~cm}$ ). The collected plant (Artemisia somai and carex spp.) was divided into eight groups, respectively, and the roots were washed with distilled water until soil-free. The plants were then put in the experimental soil. Another eight flowerpots were unplanted as control.

Soil Sample Treatment. Five grams of each Artemisia somai and carex rhizosphere soil samples which were not dried was collected after culturing for $1,2,3,6,10,16,25$, and $43 \mathrm{~d}$. Rhizosphere soil was collected by the dithering method, and the control soil was collected at the same depth. Soil samples were extracted by $50 \mathrm{~mL}$ of methanol and centrifuged at the centrifuge $(3000 \mathrm{r} / \mathrm{min})$ three times, and then the supernatant extract was filtered by quantitative paper. Rotary evaporators were used to concentrate the filtrates at a temperature of $40{ }^{\circ} \mathrm{C}$. The concentrated samples were dissolved by $4 \mathrm{~mL}$ of methanol, and filtered by $0.45 \mu \mathrm{m}$ membrane, and then analyzed for niclosamide residue and its metabolites.

Plant Sample Treatment. The Artemisia somai and carex rhizosphere samples were collected after culturing for $1,6,10$, and $16 \mathrm{~d}$, and then washed by tap water and distilled water, respectively. Samples were packed by craft paper and dried to constant weight at a temperature of $50{ }^{\circ} \mathrm{C}$. Plant samples were divided into aerial part and root part, and then ground to powder. Five grams of sample powder was dissolved in $100 \mathrm{~mL}$ of methanol, and then extracted by ultrasound and centrifuged ( $3000 \mathrm{r} / \mathrm{min}$ ) three times. The supernatant extract was filtered by quantitative paper. Rotary evaporators were used to concentrate the filtrates at the temperature of $60{ }^{\circ} \mathrm{C}$. The concentrated samples were dissolved by $4 \mathrm{~mL}$ of methanol and filtered by a
$0.45 \mu \mathrm{m}$ membrane, and then analyzed for niclosamide residues and its degradation intermediate product.

Analytical Methods. Niclosamide and its degradation intermediate product in soil and plant samples were identified by HPLC-MS/MS (Agilent 6538 Q-TOF System) equipped with an ESI source. Samples were separated on a reversed-phase column (Kromasil C18, $250 \mathrm{~mm} \times 4.6 \mathrm{~mm}$ i.d.) with a guard column $(5 \mu \mathrm{m}, 10 \mathrm{~mm} \times 4.6 \mathrm{~mm}$ i.d. $)$. Analysis conditions for the HPLC: $\mathrm{MeOH} / 0.1 \%$, methanoic acid $=70 / 30$ was used as mobile phase and flow rate was set to $0.2 \mathrm{~mL} \cdot \mathrm{min}^{-1}$ without a separation column. Full scale MS spectra both in negative modes in the mass range between 50 and $800 \mathrm{~m} / z$ were recorded.

At the same time, niclosamide and its degradation intermediate products in soil and plant samples were qualitatively and quantitatively analyzed by HPLC (2695, Waters, USA). Samples were separated on a reversed-phase column (Kromasil $\mathrm{C} 18,250 \mathrm{~mm} \times 4.6 \mathrm{~mm}$ i.d. $)$ with a guard column $(5 \mu \mathrm{m}, 10 \mathrm{~mm}$ $\times 4.6 \mathrm{~mm}$ i.d.). Mobile phase consisted of $0.2 \%$ formic acid methanol solution (A) and distilled water (B) by using a gradient program of 50:50 (A:B, v/v) in 0-4 min, 60:40 in 4-10 min, 100:0 in 10-13 $\mathrm{min}$, and 50:50 in $14-16 \mathrm{~min}$. The flow rate was $1 \mathrm{~mL} / \mathrm{min}$ and column temperature was $35^{\circ} \mathrm{C}$. A photo-diode array (PDA) detector was set at $330 \mathrm{~nm}$ for acquiring chromatograms, however, the PDA detector was set at $285 \mathrm{~nm}$ for acquiring 5-chlorinated salicylic acid chromatograms in the same chromatographic condition.

Microorganisms including bacteria, antinomycetes, and fungi, were both measured by the dilution-plate method and grew respectively in beef extract-peptone medium, antinomycetes culture medium, and rose bengal medium. The amount of microorganisms was calculated according to the following formula.

Clump count $(\mathrm{CFU}) /$ gram (dry soil) = average colony number in Petri dishes $\times$ dilution ratio/proportion of dry soil.

Calculations and Model. The degradation rate of niclosamide was calculated by fitting the data assuming firstorder kinetics. The rate constant and half-life of each sample were calculated according to

$$
C=C_{0} \mathrm{e}^{-k t}
$$

where $C$ is the concentration of niclosamide at real time, $C_{0}$ is the initial concentration of niclosamide, $k$ is the rate of degradation of niclosamide, and $t$ is the time.

\section{ASSOCIATED CONTENT}

\section{Supporting Information}

The Supporting Information is available free of charge on the ACS Publications website at DOI: 10.1021/acsomega.8b00071.

$$
\text { Calibration curves (PDF) }
$$

\section{AUTHOR INFORMATION}

\section{Corresponding Author}

*E-mail: dghuang1017@163.com. Tel.: 13479188219.

\section{ORCID}

Cui Luo: 0000-0002-7127-5090

Donggen Huang: 0000-0001-8908-1705

\section{Funding}

This work was supported by National Natural Science Foundation of China (41363005), Graduate Innovation Fund of Jiangxi Province. 


\section{Notes}

The authors declare no competing financial interest.

\section{REFERENCES}

(1) Muir, D. C. G.; Yarechewski, A. L. Degradation of Niclosamide (2',5-Dichloro-4' - nitrosalicylanilide) in Sediment and Water Systems. J. Agric. Food Chem. 1982, 30, 1028-1032.

(2) Schultz, D. P.; Harman, P. D. Hydrolysis and Photolysis of the Lampricide 2', 5-Dichloro-4'-nitrosalicylanilide (Bayer 73). Invest Fish Control 1978, 85, 1-5.

(3) Graebing, P. W.; Hubert, T. D.; Gingerich, W. H.; Chib, J. S. Aqueous photolysis of niclosamide. J. Agric. Food Chem. 2004, 52, 870878.

(4) Dai, J. R.; Cao, J. P.; Li, H. J.; Wang, W.; Liao, K.; Tao, Y. H. Studies on degradation mechanism of niclosamide ethanolamine salt I Determination of niclosamide ethanolamine salt in photolysis solution. Chin. J. Schistosomiasis Control 2009, 21, 205-208.

(5) Gonnert, R, Strufe, R. Comparative investigations of some molluscicides. In Ciba Foundation Symposium on Bilharziasis; Churchill: London, UK, 1961, 326-338.

(6) Graebing, P. W.; Chib, J. S.; Hubert, T. D.; Gingerich, W. H. Metabolism of niclosamide in sediment and water systems. J. Agric. Food Chem. 2004, 52, 5924-5932.

(7) Dawson, V. K. Environmental fate and effects of the lampricide bayluscide: A review. J. Great Lakes Res. 2003, 29, 475-492.

(8) Sakamoto, K.; Dew, W. A.; Hecnar, S. J.; Pyle, G. G. Effects of lampricide on olfaction and behavior in young-of-the-year lake sturgeon (Acipenser $\mathrm{f}$ ulvescens). Environ. Sci. Technol. 2016, 50, 3462-3468.

(9) Andrews, P.; Thyssen, J.; Lorke, D. The biology and toxicology of molluscicides, bayluscide. Pharmacol. Ther. 1983, 19, 245-295.

(10) Dai, J. R.; Wu, F.; Gao, Z. H.; Hong, Q. B. Toxicity of B002, niclosamide and their mixture against fish. Journal of Practical Parasitic Diseases 1997, 9, 168-170.

(11) SHAO, Y. The experiment of acute toxicity of the drugs to oyster. Shandong Fisheries 2010, 27, 8-9.

(12) He, M. Z.; Zhou, Y. B.; Zhou, Y.; Wang, H. Y.; Jiang, Q. W. Change of niclosamide concertration in soil through heaping and the effect of Oncomelania control. Fudan University Journal Medicine Scice 2010, 37, 16-19.

(13) El-Dib, M. A.; Aly, O. A. Persistence of some phenylamide pesticides in the aquatic environment-I hydrolysis. Water Res. 1976, 10, 1047-1050.

(14) Frank, M. P.; Graebing, P.; Chib, J. S. Effect of soil moisture and sample depth on pesticide photolysis. J. Agric. Food Chem. 2002, 50, 2607-2614.

(15) Graebing, P. W.; Frank, M. P.; Chib, J. S. Effects of fertilizer and soil components on pesticide photolysis. J. Agric. Food Chem. 2002, 50, $7332-7339$

(16) Graebing, P. W.; Chib, J. S.; Hubert, T. D.; Ginderich, W. H. Metabolism of niclosamide in sediment and water systems. J. Agric. Food Chem. 2004, 52, 5924-5932.

(17) Hamaker, J W. Decomposition: quantitative aspects. In Organic Chemical in the Solid Environment; Coring, C. A. I., Hamaker, J. W., Eds.; Dekker, New York, 1972; pp 253-340.

(18) Reregistration Eligibility Decision (RED), 3-Trifluoro-Methyl-4Nitro-Phenol and Niclosamide; USEPA, 1999: EPA 738-R-99-007.

(19) Awad, O. M. Assessment of the developmental toxicity of in Utero exposure of Wistar Albino rats to Ametryne and niclosamide. Pestic. Biochem. Physiol. 1995, 53, 1-9.

(20) Huq, F. Molecular modeling analysis of the metabolism of niclosamide. J. Pharmacol. Toxicol. 2006, 1, 376-382.

(21) Zhang, Z. H. Standards of Rational Use of Pesticides and Maximum Residue Limit; Chemical Industry Press, Beijing, 2000. 